\title{
Concevoir des interventions adaptées pour éliminer les obstacles à la vaccination
}

\author{
Katrine Habersaat ${ }^{1}$, Noni E MacDonald ${ }^{2}$, Ève Dubé ${ }^{3 *}$
}

\section{Résumé}

En dépit des efforts visant à promouvoir la vaccination et à rendre les services de vaccination facilement accessibles, les taux de couverture vaccinale demeurent inférieurs aux taux cibles de nombreux vaccins dans plusieurs administrations. Le Bureau régional de l'Organisation mondiale de la santé (OMS) pour l'Europe a élaboré l'approche des Programmes de vaccination adaptés (programmes TIP) afin d'appuyer les efforts des différents pays pour parvenir à une utilisation des vaccins élevée et équitable. Dans la présente série d'articles du Centre canadien des ressources et d'échange sur les données probantes en vaccination (CANVax), nous présentons les principaux apprentissages tirés du cadre de la planification des programmes TIP afin d'aider les planificateurs, les décideurs et les fournisseurs de vaccins à déterminer les interventions qui mèneront à une utilisation accrue du vaccin. L'approche TIP est progressive et comprend les éléments suivants : 1) une identification claire de la cause fondamentale du faible taux de vaccination; 2) une intervention fondée sur la compréhension de cette cause; et 3) une évaluation du processus de mise en œuvre et de l'impact des interventions. Au niveau des fournisseurs et patients, les approches et les idées du cadre de planification de l'approche TIP pourraient éclairer la consultation sur la vaccination en soulignant l'importance de s'engager auprès des patients et des aidants, de les écouter et de répondre à leurs besoins.
Cette oeuvre est mise à la disposition selon les termes de la licence internationale Creative Commons Attribution 4.0

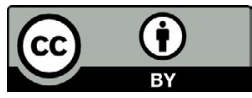

\section{Affiliations}

${ }^{1}$ Organisation mondiale de la santé en Europe, Genève, Suisse

2 Université Dalhousie, Halifax, NS

${ }^{3}$ Institut national de la santé publique du Québec, Québec, QC

\section{*Correspondance :}

eve.dube@inspq.qc.ca

Citation proposée : Habersaat K, MacDonald NE, Dubé Ė. Concevoir des interventions adaptées pour éliminer les obstacles à la vaccination. Relevé des maladies transmissibles au Canada 2021;47(3):181-5.

https://doi.org/10.14745/ccdr.v47i03a07f

Mots-clés : acceptation de vaccins, hésitation à la vaccination, interventions, évaluations, Programmes de vaccination adaptés (programmes TIP)

\section{Introduction}

En dépit des efforts visant à promouvoir la vaccination et à rendre les services de vaccination facilement accessibles, les taux de couverture vaccinale demeurent inférieurs aux taux cibles de nombreux vaccins dans plusieurs administrations. Comment pouvons-nous élaborer des interventions efficaces pour accroître l'acceptation et l'utilisation des vaccins? Cette série d'articles Edu Centre canadien des ressources et d'échange sur les données probantes et la vaccination (CANVax) présente quelques idées fondées sur l'approche des Programmes de vaccination adaptés (programmes TIP) (1). L'approche TIP a été élaborée par le Bureau régional pour l'Europe de I'Organisation mondiale de la santé (OMS) afin d'appuyer les différents pays dans leurs efforts pour parvenir à une utilisation élevée et équitable des vaccins. Le principe sous-jacent de cette approche est qu'il est nécessaire de comprendre les obstacles à la vaccination parmi les groupes de population dont la couverture est sous-optimale avant de se lancer dans des plans d'intervention. La figure 1 met en évidence les principes clés qui guident l'approche TIP. L'approche TIP est globale et progressive, et elle nécessite un investissement en temps et en ressources. Même si votre organisation n'effectue pas une évaluation TIP complète, les principales idées présentées dans cet article vous aideront à concevoir une intervention efficace pour améliorer l'acceptation et l'utilisation du vaccin.

Figure $1:$ Valeurs et principes qui guident les Programmes de vaccination adaptés

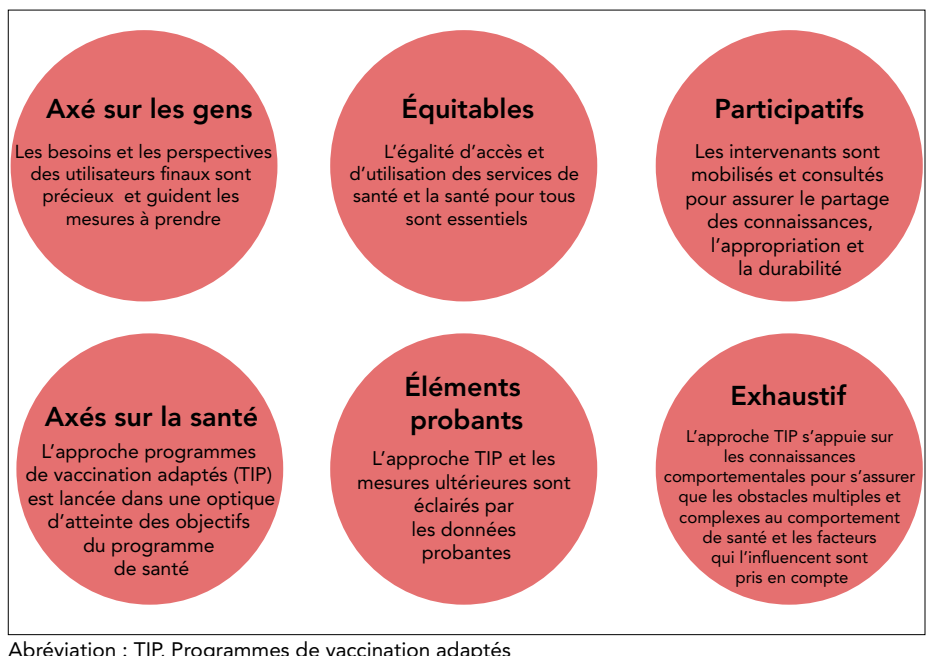

Abréviation : TIP, Programmes de vaccination adaptés 
L'approche TIP, bien qu'elle soit conçue pour être utilisée au niveau national, s'applique également au niveau des patients et fournisseurs. L'approche TIP et les apprentissages qui en découlent peuvent éclairer la planification des consultations sur les vaccins dans un bureau de fournisseurs de soins de santé.

L'objectif de ce numéro du CANVax est d'illustrer, par le biais d'une étude de cas fictive, comment les principales approches utilisées dans le cadre de planification des programmes TIP pourraient aider les planificateurs des programmes de vaccination, les décideurs et les fournisseurs de vaccins à élaborer la bonne intervention qui mènera à une augmentation de l'utilisation des vaccins.

Celui-ci est le onzième article de la série CANVAX. CANVax est une base de données en ligne qui appuie la planification et la prestation des programmes de vaccination. Cette série comprend à la fois l'identification des ressources existantes et la description des nouvelles ressources mises au point par un groupe multidisciplinaire de professionnels (2). L'article fait partie d'une série et montre comment les divers aspects relatifs à I'hésitation à face à la vaccination qui ont été considérés jusqu'à présent peuvent être appliqués pour favoriser l'acceptation du vaccin.

\section{Étude de cas canadienne}

Étude de cas, partie 1 :

Un programme de vaccination contre le virus du papillome humain (VPH) en milieu scolaire a été mis en œuvre dans votre administration en 2008. Après la première année du programme, le taux de couverture vaccinale s'est établi à plus de $80 \%$. Toutefois, au cours des années suivantes, on constate que les taux de couverture vaccinale contre le VPH ont diminué. Afin d'améliorer le taux de couverture vaccinale, une campagne de sensibilisation ciblant les parents des élèves a été mise en œuvre l'an dernier et des séances de formation ont été offertes par le personnel infirmier de l'école. Malgré ces interventions, le taux d'utilisation du vaccin contre le VPH est encore en baisse. Que peut-on faire?

L'approche TIP de l'OMS se veut une méthode pour diagnostiquer les obstacles et les facteurs qui favorisent la vaccination dans des sous-groupes spécifiques et pour concevoir des interventions pertinentes et adaptées à ces populations. L'approche TIP fait appel à des méthodes de compréhension sociale et comportementale (c'est-à-dire des méthodes de recherche axées sur les personnes et des méthodes de sciences sociales) pour concevoir et évaluer des interventions visant à modifier les comportements. Pour en apprendre davantage sur I'approche TIP, consultez la section intitulée Des programmes de vaccination adaptés (programmes TIP) (2019) (2).

La première étape du processus consiste à comprendre le problème et à en analyser les raisons afin de bien cerner le ou les obstacles.

\section{Apprentissages de l'adaptation des programmes de vaccination aux comportements : Identifier le problème; ne pas se contenter de le deviner}

Souvent, les causes d'une faible couverture vaccinale sont mal comprises, et les interventions sont conçues en fonction de l'intuition des experts plutôt que sur des données réelles.

- «Nous l'avons essayé par le passé et cela a fonctionné.»

- «Si seulement les gens savaient combien les vaccins sont sûrs et efficaces, ils choisiraient la vaccination ».

En revanche, I'approche TIP souligne que la première étape pour trouver une solution est d'avoir une bonne compréhension de la cause fondamentale du problème. Vous pouvez le faire :

- En examinant les études pertinentes menées dans votre administration

- En interrogeant les professionnels de santé de première ligne, les élèves, les parents, les bénéficiaires potentiels, les membres de la collectivité locale et/ou autres parties prenantes

L'objectif est de cerner les principaux obstacles et facteurs qui facilitent le comportement de vaccination voulu dans le groupe cible :

- Le problème est-il lié aux services de vaccination? À un manque de sensibilisation? À la désinformation sur les médias sociaux? Ce n'est qu'en ayant une bonne compréhension des causes du problème que vous serez en mesure d'élaborer une intervention efficace.

Étude de cas, partie 2 :

Des entrevues ont été menées auprès du personnel infirmier des écoles et des parents d'élèves afin d'analyser leurs opinions à l'égard du programme de vaccination contre le VPH en milieu scolaire. Les résultats ont démontré que le processus de consentement éclairé était l'un des principaux obstacles aux programmes de vaccination contre le VPH en milieu scolaire. Les parents ont indiqué ne pas savoir qu'ils devaient signer et renvoyer le formulaire au personnel infirmier de l'école pour faire vacciner leur enfant. Le personnel infirmier a fait remarquer que la courte période entre la distribution des formulaires de consentement éclairé aux élèves et le jour de vaccination les avait empêchés d'envoyer des rappels aux parents.

\section{Apprentissages de l'adaptation des programmes de vaccination aux comportements : Concevoir une intervention adaptée}

Lorsque vous avez une bonne compréhension de la cause fondamentale du problème, l'étape suivante consiste à concevoir une intervention basée à la fois sur cette compréhension et sur les ressources disponibles. Si les problèmes concernent l'accès aux services de vaccination, une intervention visant à informer les 
gens des risques et des avantages de la vaccination ne sera pas efficace. Si le manque de sensibilisation est la principale cause d'un faible taux de vaccination, il faut d'abord s'y attaquer.

En général, les interventions à composants multiples sont plus efficaces que les interventions à composant unique. Par exemple, même une intervention simple comme la modification des heures d'ouverture d'une clinique doit être communiquée au public; il n'est pas suffisant d'annoncer les nouvelles heures sur la porte de la clinique. Le modèle de la Roue du changement des comportements (voir la figure 2) peut aider à éclairer la conception de l'intervention pour aborder les comportements liés à la santé en mettant en évidence les types d'intervention pertinents, selon les obstacles et les facteurs identifiés (2). L'approche TIP a adapté ce modèle aux préoccupations liées à la vaccination (1). Notez que plusieurs composants doivent être pris en compte.

Figure 2 : Roue de changement du comportement

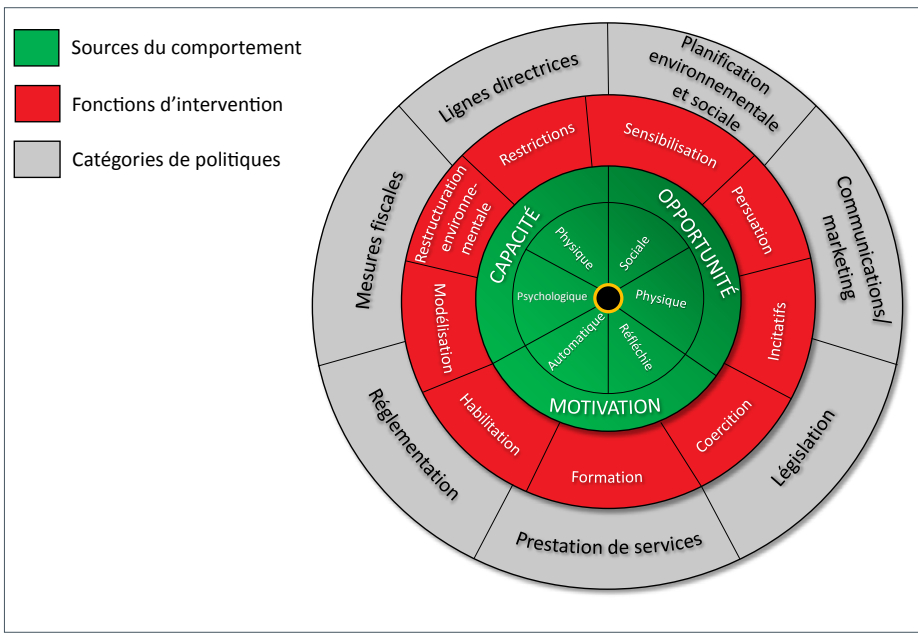

Des renseignements supplémentaires sur les interventions efficaces pour accroître l'acceptation et l'utilisation des vaccins se trouvent sur les fiches d'information CANVax (2).

Étude de cas, partie 3 :

L'évaluation qualitative a cerné les obstacles « d'opportunités " liés à l'organisation des services de vaccination.

L'intervention conçue par la suite repose sur la distribution, par les enseignants, de formulaires de consentement éclairé aux parents d'élèves au début de l'année scolaire et sur l'envoi d'un rappel par courriel un mois avant le jour de vaccination pour : 1) rappeler aux parents qu'il y aura une séance de vaccination; 2 ) leur demander de renvoyer le formulaire de consentement signé; et 3) transmettre les coordonnées du personnel infirmier de l'école au cas où les parents auraient des questions sur la vaccination. Une évaluation de la faisabilité et de l'impact de cette intervention est en cours.

\section{Apprentissages de l'adaptation des programmes de vaccination aux comportements : Mise en œuvre et évaluation}

Trop souvent, le travail visant à améliorer un programme de vaccination s'arrête après la mise en œuvre des interventions. Dans la mesure du possible, une bonne pratique consiste à évaluer le processus de mise en œuvre et l'impact des interventions. Même si vous n'effectuez pas une grande étude, essayez d'évaluer la façon dont les interventions ont été mises en œuvre et de vérifier s'il y a eu une augmentation de l'utilisation du vaccin. Cette évaluation pourrait s'effectuer par le biais des activités régulières de suivi du programme de vaccination (par exemple, l'évaluation de la couverture vaccinale avant et après la mise en œuvre de l'intervention). Cette évaluation pourrait se faire avec des sondages, des entrevues, ou simplement en discutant avec ceux qui ont participé au processus d'évaluation de la mise en œuvre jusqu'à présent, ainsi que les succès et les lacunes constatées.

\section{Adaptation des programmes de vaccination aux comportements : Approches que les fournisseurs de santé pourraient utiliser pour accroître l'utilisation des vaccins chez leurs patients et dans leur collectivité}

Le principe directeur de l'approche TIP est que, pour que la vaccination devienne une expérience possible, souhaitable et positive, il est important de s'engager auprès des patients et des aidants, de les écouter et de répondre à leurs besoins (1). Les valeurs et principes de l'approche TIP soulignent que les besoins et les perspectives de l'utilisateur final sont valorisés et guident les mesures (voir la figure 1).

\section{- Questionnez votre patient}

Le principe sous-jacent de l'approche TIP est qu'il est nécessaire de comprendre les obstacles à la vaccination. Dans le bureau des fournisseurs de soins de santé, il peut s'agir simplement d'examiner les raisons pour lesquelles le patient ou le soignant hésite à se faire vacciner. Le fait d'obtenir les véritables raisons de cette réticence aiderait le fournisseur de soins de santé à surmonter cet obstacle avec précision et efficacité. Un article précédent de la série CANVax concernant la technique d'entrevue motivationnelle a fourni des outils pratiques et des exemples de la façon dont une telle conversation pourrait se dérouler (3).

\section{- Prenez votre temps et travaillez en équipe}

L'approche TIP propose que la rencontre entre le patient et le fournisseur de soins de santé représente un moment critique dans la prise de décision en matière de vaccination. On entend souvent dire par les fournisseurs et les patients que les consultations sur la vaccination sont courtes et qu'elles ne prévoient donc que des discussions superficielles ou limitées. Toutefois, lorsque I'on applique les techniques d'entrevue 
motivationnelle (3), il est possible de fournir un counseling court et efficace concernant la vaccination. Lorsqu'il est question de patients ou de aidants très hésitants, plus de temps pourrait être requis, de sorte que les fournisseurs de soins de santé devraient prévoir passer plus de temps avec ces patients ou aidants afin d'examiner les obstacles et les facteurs favorables menant à la vaccination dans leur ensemble.

Dans de nombreuses cliniques, il y a aussi des professionnels de la santé qui sont souvent une excellente ressource, puisqu'ils peuvent prendre le temps de répondre aux questions des patients ou des aidants au sujet de la vaccination. Il est important que, dès que les patients entrent dans la clinique et rencontrent le coordonnateur, puis un infirmier ou infirmière, puis le médecin, la culture et le ton soient établis et cohérents. Si tous les fournisseurs des soins de santé sont sur la même longueur d'ondes, il est plus probable que les patients ou les aidants soient favorables aux vaccinations.

- Fournir un exemple à imiter

Une activité TIP recommandée aux fournisseurs de soins de santé est celle de de faire la démonstration de leurs comportements liés à la vaccination (e.g. confirmer qu'ils se sont fait vacciner ou qu'ils ont fait vacciner leurs enfants) et d'utiliser ces comportements à la fois pour promouvoir de bonnes pratiques de vaccination entre eux et pour donner l'exemple à leurs patients.

- $\quad$ Partagez avec vos pairs

L'approche TIP préconise un processus d'évaluation formel pour mesurer l'impact des interventions nouvellement élaborées et mises en œuvre dans le but d'accroître le taux d'utilisation des vaccins. Toutefois, une évaluation formelle n'est ni possible ni pratique dans un bureau de fournisseur de soins de santé. Par contre, il peut être extrêmement utile de faire le point, de partager vos expériences, d'identifier les obstacles spécifiques et la façon dont vous les avez traités, les stratégies qui ont fonctionné pour vous, et d'apprendre des expériences de vos collègues.

\section{Conclusion}

En conclusion, l'approche TIP est précieuse et efficace pour concevoir des interventions visant à éliminer les obstacles à la vaccination. Elle est fondée sur la compréhension des besoins et des réalités des individus et des communautés. Même si vous ne menez pas un projet formel en vous appuyant sur l'approche TIP, vous pouvez en appliquer les principes clés qui guident l'approche (figure 1) pour concevoir votre intervention (1).
Les principales approches utilisées par le cadre de planification des programmes TIP pourraient aider les planificateurs des programmes de vaccination, les décideurs politiques ainsi que les fournisseurs de vaccins à adapter les services de vaccination pour répondre aux besoins des patients et des aidants, des groupes particuliers où il est nécessaire d'augmenter l'adoption du vaccin.

\section{Déclaration des auteurs}

K. H. - Conceptualisation, rédaction et révision

N. E. M. - Validation, rédaction et révision

È. D. - Validation, rédaction de la version originale, révision et édition

Le contenu de l'article et les points de vue qui y sont exprimés n'engagent que les auteurs et ne correspondent pas nécessairement à ceux du gouvernement du Canada.

\section{Intérêts concurrents}

Aucun.

\section{Remerciement}

Les contributions au Centre canadien de ressources et d'échange sur les données probantes en vaccination (CANVax) proviennent d'un très large éventail d'auteurs, de comités, de partenaires en vaccination, d'examinateurs et surtout du secrétariat de CANVax à l'Association canadienne de santé publique.

\section{Financement}

La Dre MacDonald reçoit des subventions de l'Agence de la santé publique du Canada, des Instituts de recherche en santé du Canada, de la Régie de la santé de la Nouvelle-Écosse, du Centre de santé IWK et du Réseau canadien de recherche sur l'immunisation.

La Dre Dubé reçoit des subventions de l'Agence de santé publique du Canada, du ministère de la Santé et des Services sociaux du Québec, des Instituts de recherche en santé du Canada, du Réseau canadien de recherche sur l'immunisation et du Conseil de recherches en sciences humaines du Canada. 


\section{Références}

1. Organisation mondiale de la Santé bureau Régionale de L'Europe. Des programmes de vaccination adaptés (2019). Genève (Suisse) : OMS; 2019 (accédé 2019-10-29). http:// www.euro.who.int/en/publications/abstracts/tip-tailorin g-immunization-programmes-2019

2. Association canadienne de santé publique. Centre canadien de ressources et d'échange sur les données probantes en vaccination. Ottawa (ON) : CANVax (accédé 2019-12-15). https://www.canvax.ca/fr
3. Gagneur A. L'entrevue motivationnelle : un outil particulièrement efficace pour atténuer la réticence à la vaccination. Relevé des maladies transmissibles au Canada2020;46(4):104-9. DOI

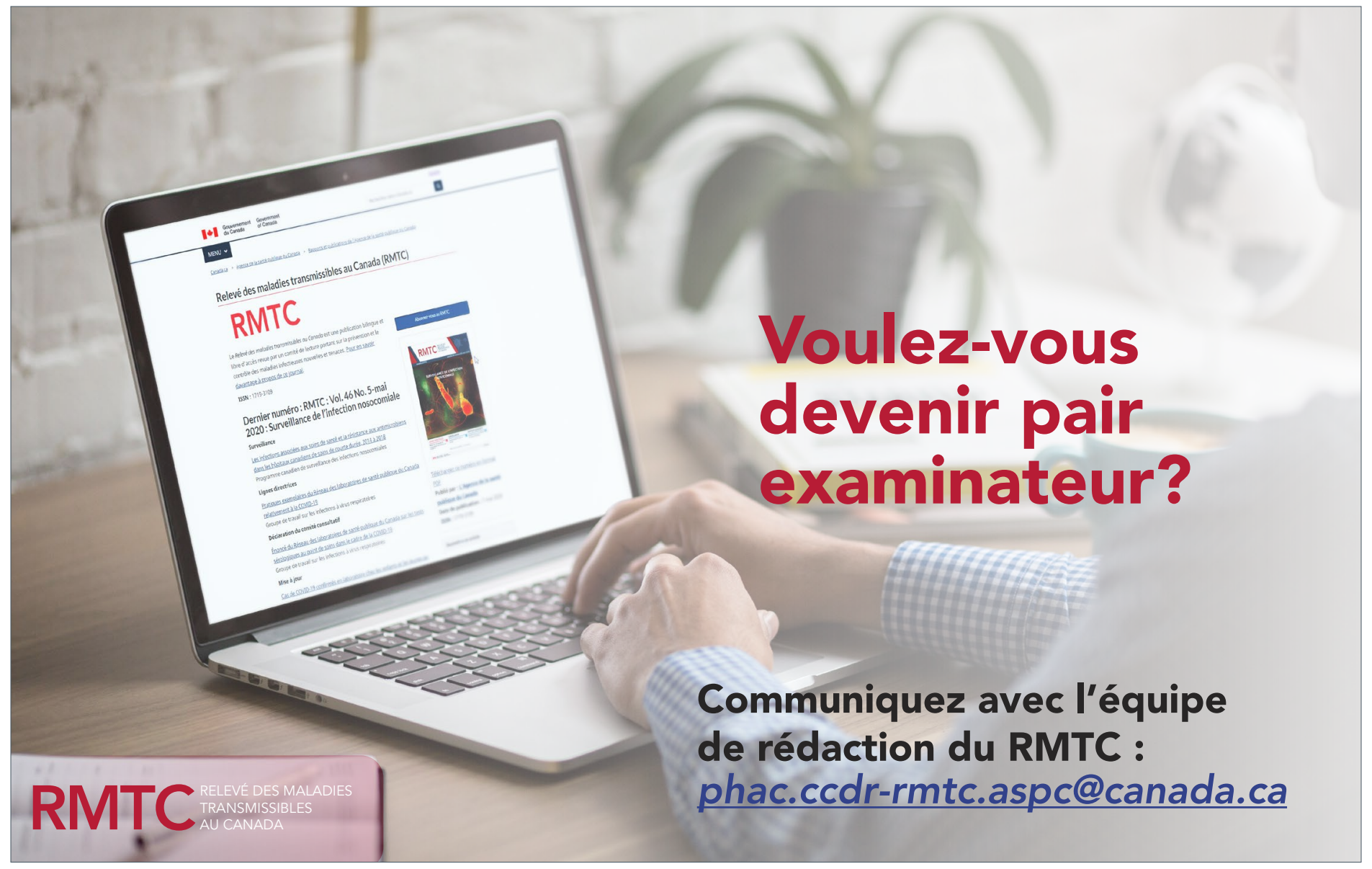

